\title{
A Modified Analytic Function Space Feynman Integral and Its Applications
}

\author{
Seung Jun Chang, Jae Gil Choi, and Hyun Soo Chung \\ Department of Mathematics, Dankook University, Cheonan 330-714, Republic of Korea \\ Correspondence should be addressed to Hyun Soo Chung; hschung@dankook.ac.kr
}

Received 12 May 2014; Accepted 3 July 2014; Published 20 July 2014

Academic Editor: Nelson Merentes

Copyright (C) 2014 Seung Jun Chang et al. This is an open access article distributed under the Creative Commons Attribution License, which permits unrestricted use, distribution, and reproduction in any medium, provided the original work is properly cited.

We analyze the generalized analytic function space Feynman integral and then defined a modified generalized analytic function space Feynman integral to explain the physical circumstances. Integration formulas involving the modified generalized analytic function space Feynman integral are established which can be applied to several classes of functionals.

\section{Introduction}

Let $C_{0}[0, T]$ denote the one-parameter Wiener space, that is, the space of continuous real-valued functions $x$ on $[0, T]$ with $x(0)=0$, and let $m$ denote Wiener measure. Since the concept of the Feynman integral was introduced by Feynman and Kac, many mathematicians studied the "analytic" Feynman integral of functionals in several classes of functionals [17]. Recently the authors have introduced an approach to the solutions of the diffusion equation and the Schrödinger equation via the Fourier-type functionals on Wiener space [6].

The function space $C_{a, b}[0, T]$, induced by a generalized Brownian motion, was introduced by Yeh in [8] and studied extensively in [9-11]. In [11] the authors have studied the generalized analytic Feynman integral for functionals in a very general function space $C_{a, b}[0, T]$.

In this paper, we present an analysis of the generalized analytic Feynman integral on function space. We define a modified generalized analytic function space Feynman integral (AFSFI) and then explain the physical circumstances with respect to an anharmonic oscillator using the concept of the modified generalized analytic Feynman integral on function space.

The Wiener process used in [1-7] is stationary in time and is free of drift while the stochastic process used in this paper, as well as in [9-12], is nonstationary in time, is subject to a drift $a(t)$, and can be used to explain the position of the Ornstein-Uhlenbeck process in an external force field [13].

\section{Preliminaries}

Let $a(t)$ be an absolutely continuous real-valued function on $[0, T]$ with $a(0)=0, a^{\prime}(t) \in L^{2}[0, T]$, and let $b(t)$ be a strictly increasing, continuously differentiable real-valued function with $b(0)=0$ and $b^{\prime}(t)>0$ for each $t \in[0, T]$. The generalized Brownian motion process $Y$ determined by $a(t)$ and $b(t)$ is a Gaussian process with mean function $a(t)$ and covariance function $r(s, t)=\min \{b(s), b(t)\}$. By Theorem 14.2 in [14], the probability measure $\mu$ induced by $Y$, taking a separable version, is supported by $C_{a, b}[0, T]$ (which is equivalent to the Banach space of continuous functions $x$ on $[0, T]$ with $x(0)=$ 0 under the sup norm). Hence, $\left(C_{a, b}[0, T], \mathscr{B}\left(C_{a, b}[0, T]\right), \mu\right)$ is the function space induced by $Y$ where $\mathscr{B}\left(C_{a, b}[0, T]\right)$ is the Borel $\sigma$-algebra of $C_{a, b}[0, T]$. We then complete this function space to obtain $\left(C_{a, b}[0, T], \mathscr{W}\left(C_{a, b}[0, T]\right), \mu\right)$ where $\mathscr{W}\left(C_{a, b}[0, T]\right)$ is the set of all Wiener measurable subsets of $C_{a, b}[0, T]$.

A subset $A$ of $C_{a, b}[0, T]$ is said to be scale-invariant measurable provided $\rho A \in \mathscr{W}\left(C_{a, b}[0, T]\right)$ for all $\rho>0$, and a scale-invariant measurable set $N$ is said to be a scale-invariant 
null set provided $\mu(\rho N)=0$ for all $\rho>0$. A property that holds except on a scale-invariant null set is said to hold scaleinvariant almost everywhere(s-a.e.) [15].

Let $L_{a, b}^{2}[0, T]$ be the Hilbert space of functions on $[0, T]$ which are Lebesgue measurable and square integrable with respect to the Lebesgue Stieltjes measures on $[0, T]$ induced by $a(\cdot)$ and $b(\cdot)$; that is,

$$
\begin{aligned}
L_{a, b}^{2}[0, T]= & \left\{v: \int_{0}^{T} v^{2}(s) d b(s)<\infty,\right. \\
& \left.\int_{0}^{T} v^{2}(s) d|a|(s)<\infty\right\},
\end{aligned}
$$

where $|a|(t)$ denotes the total variation of the function $a$ on the interval $[0, t]$.

For $u, v \in L_{a, b}^{2}[0, T]$, let

$$
(u, v)_{a, b}=\int_{0}^{T} u(t) v(t) d[b(t)+|a|(t)] .
$$

Then $(\cdot, \cdot)_{a, b}$ is an inner product on $L_{a, b}^{2}[0, T]$ and $\|u\|_{a, b}=$ $\sqrt{(u, u)_{a, b}}$ is a norm on $L_{a, b}^{2}[0, T]$. In particular note that $\|u\|_{a, b}=0$ if and only if $u(t)=0$ a.e. on $[0, T]$. Furthermore $\left(L_{a, b}^{2}[0, T],\|\cdot\|_{a, b}\right)$ is a separable Hilbert space. Note that all functions of bounded variation on $[0, T]$ are elements of $L_{a, b}^{2}[0, T]$. Also note that if $a(t) \equiv 0$ and $b(t)=t$, then $L_{a, b}^{2}[0, T]=L^{2}[0, T]$. In fact,

$$
\begin{gathered}
\left(L_{a, b}^{2}[0, T],\|\cdot\|_{a, b}\right) \subset\left(L_{0, b}^{2}[0, T],\|\cdot\|_{0, b}\right) \\
=\left(L^{2}[0, T],\|\cdot\|_{2}\right)
\end{gathered}
$$

since the two norms $\|\cdot\|_{0, b}$ and $\|\cdot\|_{2}$ are equivalent.

For $v \in L_{a, b}^{2}[0, T]$ and $x \in C_{a, b}[0, T]$ we let

$$
\langle v, x\rangle=\int_{0}^{T} v(t) d x(t)
$$

denote the Paley-Wiener-Zygmund (PWZ) stochastic integral. Following are some facts about the PWZ stochastic integral [10-12].

(1) The PWZ stochastic integral $\langle v, x\rangle$ is essentially independent of the complete orthonormal set $\left\{\phi_{j}\right\}_{j=1}^{\infty}$.

(2) If $v$ is of bounded variation on $[0, T]$, then the PWZ stochastic integral $\langle v, x\rangle$ equals the Riemann-Stieltjes integral $\int_{0}^{T} v(t) d x(t)$ for s-a.e. $x \in C_{a, b}[0, T]$.

(3) The PWZ integral has the expected linearity properties.

(4) For all $v \in L_{a, b}^{2}[0, T],\langle v, x\rangle$ is a Gaussian random variable with mean $\int_{0}^{T} v(s) d a(s)$ and variance $\int_{0}^{T} v^{2}(s)$ $d b(s)$.

Throughout this paper we will assume that each functional $F: C_{a, b}[0, T] \rightarrow \mathbb{C}$ we consider is scale-invariant measurable and that

$$
\int_{C_{a, b}[0, T]}|F(\rho x)| d \mu(x)<\infty
$$

for each $\rho>0$.

We finish this section by stating the notion of generalized analytic function space Feynman integral, cf. [10, 11].

Definition 1. Let $\mathbb{C}$ denote the complex numbers, let $\mathbb{C}_{+}=$ $\{\lambda \in \mathbb{C}: \operatorname{Re}(\lambda)>0\}$, and let $\widetilde{\mathbb{C}}_{+}=\{\lambda \in \mathbb{C}:$ $\lambda \neq 0$ and $\operatorname{Re}(\lambda) \geq 0\}$. Let $F: C_{a, b}[0, T] \rightarrow \mathbb{C}$ be a measurable functional such that, for each $\lambda>0$, the function space integral

$$
J(\lambda)=\int_{C_{a, b}[0, T]} F\left(\lambda^{-1 / 2} x\right) d \mu(x)
$$

exists. If there exists a function $J^{*}(\lambda)$ analytic in $\mathbb{C}_{+}$such that $J^{*}(\lambda)=J(\lambda)$ for all $\lambda>0$, then $J^{*}(\lambda)$ is defined to be the analytic function space integral of $F$ over $C_{a, b}[0, T]$ with parameter $\lambda$, and for $\lambda \in \mathbb{C}_{+}$we write

$$
J^{*}(\lambda)=\int_{C_{a, b}[0, T]}^{a n_{\lambda}} F(x) d \mu(x) .
$$

Let $q \neq 0$ be a real number and let $F$ be a functional such that $J^{*}(\lambda)$ exists for all $\lambda \in \mathbb{C}_{+}$. If the following limit exists, we call it the generalized AFSFI of $F$ with parameter $q$ and we write

$$
\int_{C_{a, b}[0, T]}^{a n f_{q}} F(x) d \mu(x)=\lim _{\lambda \rightarrow-i q} \int_{C_{a, b}[0, T]}^{a n_{\lambda}} F(x) d \mu(x),
$$

where $\lambda \rightarrow-i q$ through values in $\mathbb{C}_{+}$.

\section{Analogue of the Generalized AFSFI}

The differential equation

$$
\frac{\partial}{\partial t} \psi(u, t)=\frac{1}{2 \lambda} \Delta \psi(u, t)-V(u) \psi(u, t)
$$

is called the diffusion equation with initial condition $\psi(u, 0)=\varphi(u)$, where $\Delta$ is the Laplacian and $V$ is an appropriate potential function. Many mathematicians have considered the Wiener integral of functionals of the form

$$
F\left(\lambda^{-1 / 2} x+u\right)
$$

where $u$ is a real number. It is a well-known fact that the Wiener integral of the functional having the form

$$
\exp \left\{-\int_{0}^{T} V\left(\lambda^{-1 / 2} x(t)+u\right) d t\right\} \varphi\left(\lambda^{-1 / 2} x(T)+u\right)
$$

forms the solution of the diffusion equation (9) by the Feynman-Kac formula. If time is replaced by an imaginary time, this diffusion equation becomes the Schrödinger equation

$$
i \frac{\partial}{\partial t} \psi(u, t)=-\frac{1}{2} \Delta \psi(u, t)+V(u) \psi(u, t)
$$


with the initial condition $\psi(u, 0)=\varphi(u)$. Hence the solution to the Schrödinger equation (12) can be obtained via the analytic Feynman integral. An approach to finding the solution to the diffusion equation (9) and the Schrödinger equation (12) involves the harmonic oscillator $V(u)=(k / 2) u^{2}$; for a more detailed study, see [6]. However, it can be difficult to obtain the solution for the diffusion equation (9) and the Schrödinger equation (12) with respect to anharmonic oscillators.

In this paper, we consider the following functional:

$$
\begin{aligned}
& \exp \left\{-\int_{0}^{T} V\left(\lambda^{-1 / 2} x(t)+c(\lambda) h(t)\right) d t\right\} \\
& \times \varphi\left(\lambda^{-1 / 2} x(T)+c(\lambda) h(t)\right),
\end{aligned}
$$

where $c(\lambda)$ is a real number with respect to $\lambda$ and $h(t)$ is a realvalued function on $[0, T]$. When $h(t)=u$ for all $t \in[0, T]$ and $c(\lambda)$ is independent of the value $\lambda$, the functional in (13) reduces the functional in (11). That is to say, our functional (13) is more generalized compared with the functional in (11). Hence, all results and formulas for the functional in (11) are special cases of our results and formulas.

We will now explain the importance of the functionals given by (13). For a positive real number $k$, when the potential function is $V(u)=(k / 2) u^{2}$, the diffusion equation (9) is called the diffusion equation for a harmonic oscillator with $V$. For $\xi \in \mathbb{R}$

$$
V_{1}(u) \equiv V(u+\xi)=\frac{k}{2}(u+\xi)^{2}
$$

is just the translation of $V$; thus, it is called the diffusion equation for a harmonic oscillator with $V_{1}$. However, for an appropriate function $h(t)$ on $[0, T]$,

$$
V_{2}(u) \equiv V(u+h(u))=\frac{k}{2}(u+h(u))^{2}
$$

may be an anharmonic oscillator. For example, consider the following.

(1) If $h(t)=u^{2}$ on $[0, T]$, then

$$
V_{3}(u)=\frac{k}{2}\left(u^{2}+2 u^{3}+u^{4}\right) \text {. }
$$

In this case, the diffusion equation (9) is called the diffusion equation for anharmonic oscillator with $V_{3}$ because it contains the " $u^{3}$-term." This means that the status of the harmonic oscillator can be exchanged for the status of the anharmonic oscillator under certain physical circumstances. We can explain this phenomenon by considering the Wiener integral of the functional in (13).

(2) For a real number $\gamma$, if $h(t)=-u+\sqrt{u^{2}\left(u^{2}-\gamma^{2}\right)}$ on $[0, T]$, then

$$
V_{4}(u)=\frac{k}{2} u^{2}\left(u^{2}-\gamma^{2}\right) .
$$

In this case, the diffusion equation (9) is called the diffusion equation for double-well potential with $V_{4}$. As such, it is a harmonic oscillator.
(3) Furthermore, we see that, for $v \in L_{a, b}^{2}[0, T], h \in$ $C_{a, b}[0, T]$, and $u \in \mathbb{R}$,

$$
\begin{gathered}
\langle v, x+u\rangle=\langle v, x\rangle, \\
\langle v, x+h\rangle=\langle v, x\rangle+\langle v, h\rangle
\end{gathered}
$$

provided $\langle v, h\rangle \neq 0$. Thus, the functionals presented in this paper are more meaningful than the functionals given in previous papers $[6,11]$. This also has implications regarding the generalizations of our research observations.

We are now ready to state the definition of the modified generalized AFSFI.

Definition 2. Let $h \in C_{a, b}[0, T]$ be given. Let $F: C_{a, b}[0, T] \rightarrow$ $\mathbb{C}$ be such that, for each $\lambda>0$, the function space integral

$$
J(\lambda)=\int_{C_{a, b}[0, T]} F\left(\lambda^{-1 / 2} x+c(\lambda) h\right) d \mu(x)
$$

exists for all $\lambda>0$ where $c(\lambda)$ is a nonnegative real number which depends on $\lambda$. If there exists a function $J^{*}(\lambda)$ analytic in $\mathbb{C}_{+}$such that $J^{*}(\lambda)=J(\lambda)$ for all $\lambda>0$, then $J^{*}(\lambda)$ is defined to be the modified analytic function space integral of $F$ over $C_{a, b}[0, T]$ with parameter $\lambda$, and for $\lambda \in \mathbb{C}_{+}$we write

$$
J^{*}(\lambda)=\int_{C_{a, b}[0, T]}^{a n_{\lambda}^{c(\lambda)}, h} F(x) d \mu(x) .
$$

Let $q \neq 0$ be a real number and let $F$ be a functional such that $\int_{C_{a, b}[0, T]}^{a n_{\lambda}^{c(\lambda)}, h} F(x) d \mu(x)$ exists for all $\lambda \in \mathbb{C}_{+}$. If the following limit exists, we call it the modified generalized AFSFI of $F$ with parameter $q$ and we write

$$
\int_{C_{a, b}[0, T]}^{a n f_{q}^{c(q)}, h} F(x) d \mu(x)=\lim _{\lambda \rightarrow-i q} \int_{C_{a, b}[0, T]}^{a n_{\lambda}^{c(\lambda)}, h} F(x) d \mu(x),
$$

where $\lambda$ approaches $-i q$ through values in $\mathbb{C}_{+}$.

Remark 3. We have the following assertions with respect to the modified generalized AFSFI.

(1) If $h(t) \equiv 0$ on $[0, T]$ or $c(\lambda)=0$, then we can write

$$
\begin{aligned}
& \int_{C_{a, b}[0, T]}^{a n_{\lambda}^{c(\lambda)}, h} F(x) d \mu(x)=\int_{C_{a, b}[0, T]}^{a n_{\lambda}} F(x) d \mu(x), \\
& \int_{C_{a, b}[0, T]}^{a n f_{q}^{c(q)}, h} F(x) d \mu(x)=\int_{C_{a, b}[0, T]}^{a n f_{q}} F(x) d \mu(x) .
\end{aligned}
$$

(2) In the setting of classical Wiener space (in our research, when $a(t) \equiv 0$ and $b(t)=t$ on $[0, T])$, our modified generalized AFSFI, the generalized AFSFI, and the analytic Feynman integral coincide. Hence all results and formulas in $[2,3,5,6,16]$ are corollaries of our results and formulas in this paper.

We conclude this section by listing several integration formulas for simple functionals to compare with the generalized AFSFI and the modified generalized AFSFI. For all nonzero real number $q$, we have Tables 1 and 2 . 
TABLE 1: Modified generalized AFSFI $(j=1,2)$.

\begin{tabular}{cc}
\hline & $\int_{C_{a, b}[0, T]}^{a n f_{q}^{c(q)}, h} F_{j}(x) d \mu(x)$ \\
\hline$F_{1}(x)=x(T)$ & $\left(\frac{i}{q}\right)^{1 / 2} a(T)+c(q) h(T)$ \\
\hline$F_{2}(x)=e^{x(T)}$ & $\exp \left\{\left(\frac{i}{q}\right)^{1 / 2} a(T)+\frac{i}{2 q} b(T)+c(q) h(T)\right\}$ \\
\hline
\end{tabular}

TABLE 2: Generalized AFSFI $(j=1,2)$.

\begin{tabular}{lr}
\hline$F_{1}(x)=x(T)$ & $\int_{C_{a, b}[0, T]}^{a n f} F_{j}(x) d \mu(x)$ \\
\hline$F_{2}(x)=e^{x(T)}$ & $\exp \left\{\left(\frac{i}{q}\right)^{1 / 2} a(T)\right.$ \\
\hline
\end{tabular}

\section{Some Properties for the Modified Generalized AFSFI}

In this section we establish a Fubini theorem for the modified analytic function space integrals and the modified generalized AFSFIs for functionals on $C_{a, b}[0, T]$. We also use these Fubini theorems to establish various modified generalized analytic Feynman integration formulas.

First, we define a function to simply express many results and formulas in this paper. For $n \geq 2$, define a function $H_{n}$ : $\widetilde{\mathbb{C}}_{+}^{n} \rightarrow \widetilde{\mathbb{C}}_{+}$by

$$
H_{n}\left(z_{1}, \ldots, z_{n}\right)=\sum_{j=1}^{n} z_{j}^{-1 / 2}-\left(\sum_{j=1}^{n} z_{j}^{-1}\right)^{1 / 2}
$$

where $\sum_{j=1}^{n} z_{j}^{-1 / 2} \neq 0$ and $\sum_{j=1}^{n} z_{j}^{-1} \neq 0$. Note that $H_{n}$ is a symmetric function for all $n=2,3, \ldots$. In this paper we will assume that, for all $\left(z_{1}, \ldots, z_{n}\right) \in \widetilde{\mathbb{C}}_{+}^{n}$ and $\left(\sum_{j=1}^{n} z_{j}^{-1}\right)^{1 / 2}$, $n=1,2, \ldots$, and $z_{j}^{-1 / 2}, j=1,2, \ldots, n$, are always chosen to have positive real parts.

In our first theorem, we show that the modified generalized AFSFIs are commutative.

Theorem 4. Let $h_{1}$ and $h_{2}$ be elements of $C_{a, b}[0, T]$ and let $F$ be a functional defined on $C_{a, b}[0, T]$ such that

$$
\begin{aligned}
& \int_{C_{a, b}^{2}[0, T]}\left|F\left(\gamma x+\beta y+c(\gamma) h_{1}+c(\beta) h_{2}\right)\right| \\
& \quad \times d(\mu \times \mu)(x, y)<\infty
\end{aligned}
$$

for all nonzero real numbers $\gamma$ and $\beta$. Then for all $q_{1}, q_{2} \in \mathbb{R}-$ $\{0\}$,

$$
\begin{aligned}
& \int_{C_{a, b}[0, T]}^{a n f_{q_{2}}^{c\left(q_{2}\right)}, h_{2}}\left(\int_{C_{a, b}[0, T]}^{a n f_{q_{1}}^{c\left(q_{1}\right)}, h_{1}} F(x+y) d \mu(x)\right) d \mu(y) \\
& \quad \doteq \int_{C_{a, b}[0, T]}^{a n f_{q_{1}}^{c\left(q_{1}\right)}, h_{1}}\left(\int_{C_{a, b}[0, T]}^{a n f_{q_{2}}^{c\left(q_{2}\right)}, h_{2}} F(x+y) d \mu(y)\right) d \mu(x),
\end{aligned}
$$

where $\doteq$ means that if either side exists, both sides exist and equality holds.

Proof. First, using the symmetric property, for all $\lambda, \beta>0$,

$$
\begin{gathered}
\int_{C_{a, b}^{2}[0, T]} F\left(\lambda^{-1 / 2} x+\beta^{-1 / 2} y+c(\lambda) h_{1}\right. \\
\left.+c(\beta) h_{2}\right) d(\mu \times \mu)(x, y) \\
=\int_{C_{a, b}^{2}[0, T]} F\left(\beta^{-1 / 2} y+\lambda^{-1 / 2} x+c(\beta) h_{2}\right. \\
\left.+c(\lambda) h_{1}\right) d(\mu \times \mu)(y, x) .
\end{gathered}
$$

This can be analytically continued in $\lambda$ and $\beta$ for $(\lambda, \beta)$ and so we have, for all $(\lambda, \beta) \in \mathbb{C}_{+} \times \mathbb{C}_{+}$,

$$
\begin{aligned}
\int_{C_{a, b}[0, T]}^{a n_{\beta}^{c(\beta)}, h_{2}}\left(\int_{C_{a, b}[0, T]}^{a n_{\lambda}^{c(\lambda)}, h_{1}} F(x+y) d \mu(x)\right) d \mu(y) \\
\quad \doteq \int_{C_{a, b}[0, T]}^{a n_{\lambda}^{c(\lambda)}, h_{1}}\left(\int_{C_{a, b}[0, T]}^{a n_{\beta}^{c(\beta)}, h_{2}} F(x+y) d \mu(y)\right) d \mu(x) .
\end{aligned}
$$

Next, let $E$ be a subset of $\widetilde{\mathbb{C}}_{+} \times \widetilde{\mathbb{C}}_{+}$containing the point $\left(-i q_{1},-i q_{2}\right)$ and it is such that $(\lambda, \beta) \in E$ implies that $\lambda+\beta \neq 0$. Note that the function

$$
\mathscr{H}(\lambda, \beta) \equiv \int_{C_{a, b}[0, T]}^{a n_{\beta}^{c(\beta)}, h_{2}}\left(\int_{C_{a, b}[0, T]}^{a n_{\lambda}^{c(\lambda)}, h_{1}} F(y+z) d \mu(y)\right) d \mu(z)
$$

is continuous on $E$ and is uniformly continuous on $E$ provided $E$ is compact. Then by the continuity of $\mathscr{H}$ and (27), we can establish (25) as desired.

The following theorem was established in [12, 17]. Formula (29) is called the Fubini theorem with respect to the function space integrals.

Theorem 5. Let $F$ be as in Theorem 4 above. Then

$$
\begin{aligned}
& \int_{C_{a, b}^{2}[0, T]} F(\gamma x+\beta y) d(\mu \times \mu)(x, y) \\
& =\int_{C_{a, b}[0, T]} F\left(\sqrt{\gamma^{2}+\beta^{2}} z\right. \\
& \left.\quad+\left(\gamma+\beta-\sqrt{\gamma^{2}+\beta^{2}}\right) a\right) d \mu(z) \\
& =\int_{C_{a, b}[0, T]} F\left(\sqrt{\gamma^{2}+\beta^{2}} z+H_{2}\left(\gamma^{-2}, \beta^{-2}\right) a\right) d \mu(z) .
\end{aligned}
$$


To establish Theorem 7, we need the following lemma.

Lemma 6. Let $F$ be as in Theorem 4 above. Then for all $(\lambda, \beta) \in$ $\mathbb{C}_{+} \times \mathbb{C}_{+}$with $\lambda+\beta \neq 0$,

$$
\begin{gathered}
\int_{C_{a, b}[0, T]}^{a n_{\beta}}\left(\int_{C_{a, b}[0, T]}^{a n_{\lambda}} F(x+y) d \mu(x)\right) d \mu(y) \\
\quad \doteq \int_{C_{a, b}[0, T]}^{a n_{\gamma}^{c(\gamma)}, a} F(z) d \mu(z),
\end{gathered}
$$

where $\gamma=\lambda \beta /(\lambda+\beta)$ and $c(\gamma)=H_{2}(\lambda, \beta)$.

Proof. Using (29), it follows that for $\lambda>0$ and $\beta>0$

$$
\begin{aligned}
& \int_{C_{a, b}^{2}[0, T]} F\left(\lambda^{-1 / 2} x+\beta^{-1 / 2} y\right) d(\mu \times \mu)(x, y) \\
& =\int_{C_{a, b}[0, T]} F\left(\sqrt{\lambda^{-1}+\beta^{-1}} z+H_{2}(\lambda, \beta) a\right) d \mu(z) .
\end{aligned}
$$

This last expression is defined for $\lambda>0$ and $\beta>0$. For $\beta>0$, it can be analytically continued in $\lambda \in \mathbb{C}_{+}$. Also for $\lambda>0$, it can be analytically continued in $\beta \in \mathbb{C}_{+}$. Therefore since $\lambda \in \mathbb{C}_{+}$and $\beta \in \mathbb{C}_{+}$implies that $\lambda \beta /(\lambda+\beta) \in \mathbb{C}_{+}$, we conclude that the last expression in proof of Lemma 6 can be analytically continued into $\mathbb{C}_{+}$to equal the analytic function space integral

$$
\int_{C_{a, b}[0, T]}^{a n_{\gamma}^{c(\gamma)}, a} F(z) d \mu(z)
$$

which completes the proof of Lemma 6 as desired.

The following theorem is the main result with respect to the modified generalized AFSFI.

Theorem 7. Let $F$ be as in Lemma 6 above. Then for all $q_{1}, q_{2} \in$ $\mathbb{R}-\{0\}$ with $q_{1}+q_{2} \neq 0$,

$$
\begin{gathered}
\int_{C_{a, b}[0, T]}^{a n f}\left(\int_{C_{a, b}[0, T]}^{a n f_{q_{1}}} F(x+y) d \mu(x)\right) d \mu(y) \\
\quad \doteq \int_{C_{a, b}[0, T]}^{a n f_{q_{3}}^{c\left(q_{3}\right)}, a} F(z) d \mu(z),
\end{gathered}
$$

where $q_{3}=q_{1} q_{2} /\left(q_{1}+q_{2}\right)$ and $c\left(q_{3}\right)=H_{2}\left(-i q_{1},-i q_{2}\right)$.

Proof. First note that, for all $q_{1}, q_{2} \in \mathbb{R}-\{0\}$ with $q_{1}+q_{2} \neq 0$, if $\lambda \rightarrow-i q_{1}$ and $\beta \rightarrow-i q_{2}$, then $\lambda \beta /(\lambda+\beta) \rightarrow-i\left(q_{1} q_{2} /\left(q_{1}+\right.\right.$ $\left.q_{2}\right)$ ). Now using this fact and (30) it follows that

$$
\begin{aligned}
\int_{C_{a, b}[0, T]}^{a n f} f_{q_{2}} & \left(\int_{C_{a, b}[0, T]}^{a n f_{q_{1}}} F(x+y) d \mu(x)\right) d \mu(y) \\
& \doteq \lim _{\beta \rightarrow-i q_{2} \lambda} \lim _{\lambda \rightarrow-i q_{1}} \int_{C_{a, b}[0, T]}^{a n_{\gamma}^{c(\gamma)}, a} F(z) d \mu(z) \\
& \doteq \lim _{\lambda \beta /(\lambda+\beta) \rightarrow-i\left(q_{1} q_{2} /\left(q_{1}+q_{2}\right)\right)} \int_{C_{a, b}[0, T]}^{a n_{\gamma}^{c(\gamma)}, a} F(z) d \mu(z) \\
& \doteq \int_{C_{a, b}[0, T]}^{a n f_{q_{3}}^{c\left(q_{3}\right)}, a} F(z) d \mu(z),
\end{aligned}
$$

where $\gamma$ and $c(\gamma)$ are as in Lemma 6. Hence we complete the proof as desired.

Equations (35)-(37) below follow by mathematical induction and Theorem 7 above.

Corollary 8. Let $F$ be as in Theorem 7 above. Then one has the following assertions.

(1) For all $q \in \mathbb{R}-\{0\}$,

$$
\begin{gathered}
\int_{C_{a, b}[0, T]}^{a n f_{q}}\left(\int_{C_{a, b}[0, T]}^{a n f_{q}} F(x+y) d \mu(x)\right) d \mu(y) \\
\quad \doteq \int_{C_{a, b}[0, T]}^{a n f_{q / 2}^{c(q)}, a} F(z) d \mu(z),
\end{gathered}
$$

where $c(q)=H_{2}(-i q,-i q)$.

(2) For all $q_{1}, \ldots, q_{n} \in \mathbb{R}-\{0\}$ with $\sum_{j=1}^{k}\left(q_{1} \ldots q_{k} / q_{j}\right) \neq 0$ for $k=2, \ldots, n$,

$\int_{C_{a, b}[0, T]}^{a n f_{q_{n}}} \cdots \int_{C_{a, b}[0, T]}^{a n f_{q_{1}}} F\left(x_{1}+\cdots+x_{n}\right) d(\mu \times \cdots \times \mu)(\vec{x})$

$$
\doteq \int_{C_{a, b}[0, T]}^{a n f_{\beta_{n}}^{c\left(\beta_{n}\right)}, a} F(z) d \mu(z),
$$

where $\vec{x}=\left(x_{1}, \ldots, x_{n}\right), \beta_{n}=q_{1} \ldots q_{n} / \sum_{j=1}^{n}\left(q_{1} \ldots q_{n} /\right.$ $\left.q_{j}\right)$, and $c\left(\beta_{n}\right)=H_{n}\left(-i q_{1}, \ldots,-i q_{n}\right)$. Furthermore,

$$
\begin{aligned}
\int_{C_{a, b}[0, T]}^{a n f_{q}} & \cdots \int_{C_{a, b}[0, T]}^{a n f_{q}} F\left(x_{1}+\cdots+x_{n}\right) d(\mu \times \cdots \times \mu)(\vec{x}) \\
& \doteq \int_{C_{a, b}[0, T]}^{a n f_{q / n}^{c(q / n)}, a} F(z) d \mu(z),
\end{aligned}
$$

where $c(q / n)=H_{2}(-i q, \ldots,-i q)$.

Next we establish some integration formulas with respect to the modified generalized AFSFIs.

(1) A formula showing that the double modified generalized AFSFIs can be expressed by just one modified 
generalized AFSFI. For all $q_{1}, q_{2} \in \mathbb{R}-\{0\}$ with $q_{1}+q_{2} \neq 0$,

$$
\begin{gathered}
\int_{C_{a, b}[0, T]}^{a n f c_{q_{2}}^{c\left(q_{2}\right)}, a}\left(\int_{C_{a, b}[0, T]}^{a n f_{q_{1}}^{c\left(q_{1}\right)}, a} F(x+y) d \mu(x)\right) d \mu(y) \\
\quad=\int_{C_{a, b}[0, T]}^{a n f_{q_{1} q_{2} /\left(q_{1}+q_{2}\right)}^{c\left(q_{1}, q_{2}\right), a}} F(z) d \mu(z),
\end{gathered}
$$

where $c\left(q_{1}, q_{2}\right)=H_{2}\left(-i q_{1},-i q_{2}\right)+c\left(q_{1}\right)+c\left(q_{2}\right)$. Furthermore, if $c\left(q_{1}\right)+c\left(q_{2}\right)=-H_{2}\left(-i q_{1},-i q_{2}\right)$, then

$$
\begin{aligned}
& \int_{C_{a, b}[0, T]}^{a n f_{q_{2}}^{c\left(q_{2}\right)}, a}\left(\int_{C_{a, b}[0, T]}^{a n f_{q_{1}}^{c\left(q_{1}\right)}, a} F(x+y) d \mu(x)\right) d \mu(y) \\
& \doteq \int_{C_{a, b}[0, T]}^{a n f} f_{q_{1} q_{2} /\left(q_{1}+q_{2}\right)} F(z) d \mu(z) \text {. }
\end{aligned}
$$

(2) A relationship between the modified generalized AFSFI and the generalized AFSFI. For all $q_{1}, q_{2} \in$ $\mathbb{R}-\{0\}$ with $q_{1}+q_{2} \neq 0$,

$$
\begin{gathered}
\int_{C_{a, b}[0, T]}^{a n f f_{q_{1}}^{\left(c q_{1}\right)}, a}\left(\int_{C_{a, b}[0, T]}^{a n f_{q_{2}}} F(x+y) d \mu(x)\right) d \mu(y) \\
\quad=\int_{C_{a, b}[0, T]}^{a n f_{q_{3}}^{c\left(q_{3}\right)}, a} F(z) d \mu(z),
\end{gathered}
$$

where $q_{3}=q_{1} q_{2} /\left(q_{1}+q_{2}\right)$ and $c\left(q_{3}\right)=H_{2}\left(-i q_{1},-i q_{2}\right)+$ $c\left(q_{1}\right)$.

(3) A formula relating the modified generalized AFSFI and the generalized AFSFI. For all $q_{1}, q_{2} \in \mathbb{R}-\{0\}$,

$$
\begin{aligned}
& \int_{C_{a, b}[0, T]}^{a n f_{q_{1}}^{c\left(q_{1}\right)}, a}\left(\int_{C_{a, b}[0, T]}^{a n f_{q_{2}}} F(x+y) d \mu(x)\right) d \mu(y) \\
& \quad \doteq \int_{C_{a, b}[0, T]}^{a n f_{q_{2}}}\left(\int_{C_{a, b}[0, T]}^{a n f q_{q_{1}}^{\left(c q_{1}\right)}, a} F(x+y) d \mu(x)\right) d \mu(y) .
\end{aligned}
$$

\section{Examples}

In this section, we provide several brief examples in which we apply our formulas and results.

5.1. Banach Algebra $\delta\left(L_{a, b}^{2}[0, T]\right)$. Let $M\left(L_{a, b}^{2}[0, T]\right)$ be the space of complex-valued, countably additive Borel measures on $L_{a, b}^{2}[0, T]$. The Banach algebra $\mathcal{S}\left(L_{a, b}^{2}[0, T]\right)$ consists of those functionals $F$ on $C_{a, b}[0, T]$ expressible in the form

$$
F(x)=\int_{L_{a, b}^{2}[0, T]} \exp \{i\langle v, x\rangle\} d f(v)
$$

for s-a.e. $x \in C_{a, b}[0, T]$ where the associated measure $f$ is an element of $M\left(L_{a, b}^{2}[0, T]\right)$.
Example 1. Let $q_{0}$ be a fixed nonzero real number. Let $F \in \mathcal{S}\left(L_{a, b}^{2}[0, T]\right)$ be given by (42) above. Suppose that corresponding measure $f$ of $F$ satisfies the condition

$$
\int_{L_{a, b}^{2}[0, T]} \exp \left\{\frac{4}{\sqrt{2\left|q_{0}\right|}} \int_{0}^{T}|v(s)| d|a|(s)\right\}|d f(v)|<\infty .
$$

Then for all nonzero real number $q$ with $|q| \geq\left|q_{0}\right|$,

$$
\begin{aligned}
\int_{C_{a, b}[0, T]}^{a n f_{q}^{c(q)}, a} F(x) d \mu(x) & \\
=\int_{L_{a, b}^{2}[0, T]} \exp \{- & \frac{i}{2 q}\left(v^{2}, b^{\prime}\right) \\
+ & \left.+i\left(c(q)+\left(\frac{i}{q}\right)^{1 / 2}\right)\left(v, a^{\prime}\right)\right\} d f(v),
\end{aligned}
$$

where

$$
\left(v, a^{\prime}\right)=\int_{0}^{T} v(t) d a(t), \quad\left(v^{2}, b^{\prime}\right)=\int_{0}^{T} v^{2}(t) d b(t) .
$$

Next, using Theorem 7, we can compute the double generalized AFSFIs of $F \in \mathcal{S}\left(L_{a, b}^{2}[0, T]\right)$ by just one modified generalized AFSFI. That is to say, for all $q_{1}, q_{2} \in \mathbb{R}$ with $\left|q_{1}\right| \geq\left|q_{0}\right|,\left|q_{2}\right| \geq\left|q_{0}\right|$ and $q_{1}+q_{2} \neq 0$,

$$
\begin{gathered}
\int_{C_{a, b}[0, T]}^{a n f_{q_{2}}}\left(\int_{C_{a, b}[0, T]}^{a n f_{q_{1}}} F(x+y) d \mu(x)\right) d \mu(y) \\
=\int_{C_{a, b}[0, T]}^{a n f_{y}^{c(v)}, a} F(z) d \mu(z) \\
=\int_{L_{a, b}^{2}[0, T]} \exp \left\{-\frac{i}{2}\left(\frac{1}{q_{1}}+\frac{1}{q_{2}}\right)\left(v^{2}, b^{\prime}\right)\right. \\
+i\left(\left(\frac{i}{q_{1}}\right)^{1 / 2}+\left(\frac{i}{q_{2}}\right)^{1 / 2}\right) \\
\left.\times\left(v, a^{\prime}\right)\right\} d f(v),
\end{gathered}
$$

where $\gamma=q_{1} q_{2} /\left(q_{1}+q_{2}\right)$ and $c(\gamma)=H_{2}\left(-i q_{1},-i q_{2}\right)$. Furthermore the last expression in (46) equals the expression

$$
\int_{C_{a, b}[0, T]}^{a n f}\left(\int_{C_{a, b}[0, T]}^{a n f} F(x+y) d \mu(y)\right) d \mu(x) \text {. }
$$

5.2. The Fourier Transform of a Complex-Valued Measure. For given $\vec{m}=\left(m_{1}, \ldots, m_{n}\right) \in \mathbb{R}^{n}$ and $\overrightarrow{\sigma^{2}}=\left(\sigma_{1}^{2}, \ldots, \sigma_{n}^{2}\right) \in \mathbb{R}^{n}$ 
with $\sigma_{j}^{2}>0, j=1, \ldots, n$, let $\nu_{\vec{m}, \overrightarrow{\sigma^{2}}}$ be the Gaussian measure given by

$$
\nu_{\vec{m}, \overrightarrow{\sigma^{2}}}(B)=\left(\prod_{j=1}^{n} 2 \pi \sigma_{j}^{2}\right)^{-1 / 2} \int_{B} \exp \left\{-\sum_{j=1}^{n} \frac{\left(u_{j}-m_{j}\right)^{2}}{2 \sigma_{j}^{2}}\right\} d \vec{u},
$$

where $B \in \mathscr{B}\left(\mathbb{R}^{n}\right)$. Then $\nu_{\vec{m}, \overrightarrow{\sigma^{2}}}$ is a complex-valued Borel measure on $\mathbb{R}^{n}$ and

$$
\widehat{\nu_{\vec{m}, \sigma^{2}}}(\vec{u})=\exp \left\{-\frac{1}{2} \sum_{j=1}^{n} \sigma_{j}^{2} u_{j}^{2}+i \sum_{j=1}^{n} m_{j} u_{j}\right\},
$$

where $\widehat{\nu_{\vec{m}, \overrightarrow{\sigma^{2}}}}$ is the Fourier transform of the Gaussian measure $\nu_{\vec{m}, \overrightarrow{\sigma^{2}}}$.

Example 2. Let $\left\{\alpha_{1}, \ldots, \alpha_{n}\right\}$ be any orthonormal set in $L_{a, b}^{2}[0, T]$ and let $F: C_{a, b}[0, T] \rightarrow \mathbb{R}^{n}$ be the functional defined by

$$
F(x)=\widehat{\nu_{\vec{m}, \vec{\sigma}^{2}}}\left(\left\langle\alpha_{1}, x\right\rangle, \ldots,\left\langle\alpha_{n}, x\right\rangle\right),
$$

where $\operatorname{Var}\left[\left\langle\alpha_{j}, x\right\rangle^{2}\right]=1$ for all $j=1,2, \ldots, n$. Then for all nonzero real number $q$,

$$
\begin{aligned}
& \int_{C_{a, b}[0, T]}^{a n f_{q}^{c(q)}, a} F(x) d \mu(x) \\
&=\left(\prod_{j=1}^{n} \frac{1}{2\left(1-(-i q)^{-1 / 2} \sigma_{j}^{2}\right)}\right)^{1 / 2} \\
& \times \exp \left\{\sum _ { j = 1 } ^ { n } \left((-i q)^{-1 / 2}\right.\right. \\
& \times \exp \left\{-\frac{c(q)}{2} \sum_{j=1}^{n} \sigma_{j}^{2}\left\langle\alpha_{j}, h\right\rangle+i c(q) \sum_{j=1}^{n} m_{j}\left\langle\alpha_{j}, h\right\rangle\right\},
\end{aligned}
$$

where $A_{j}=\int_{0}^{T} \alpha_{j}(t) d a(t)$. Using Theorem 7, we can compute the double generalized AFSFIs of $F$ given by (50) by just one modified generalized AFSFI. That is to say, for all $q_{1}, q_{2} \in \mathbb{R}$ with $q_{1}+q_{2} \neq 0$,

$$
\begin{aligned}
& \int_{C_{a, b}[0, T]}^{a n f_{q_{2}}}\left(\int_{C_{a, b}[0, T]}^{a n f_{q_{1}}} F(x+y) d \mu(x)\right) d \mu(y) \\
& =\left(\prod_{j=1}^{n} \frac{1}{2\left(1-(-i Q)^{-1 / 2} \sigma_{j}^{2}\right)}\right)^{1 / 2} \\
& \times \exp \left\{\sum _ { j = 1 } ^ { n } \left((-i Q)^{-1 / 2}\right.\right. \\
& \left.\times\left[i 4 A_{j} m_{j}-(-i Q)^{-1 / 2} m_{j}^{2}+2 A_{j}^{2} \sigma_{j}^{2}\right]\right) \\
& \left.\times\left(2\left(2-(-i Q)^{-1 / 2} \sigma_{j}^{2}\right)\right)^{-1}\right\} \\
& \times \exp \left\{-\frac{c(Q)}{2} \sum_{j=1}^{n} \sigma_{j}^{2}\left\langle\alpha_{j}, h\right\rangle+i c(Q) \sum_{j=1}^{n} m_{j}\left\langle\alpha_{j}, h\right\rangle\right\},
\end{aligned}
$$

where $Q=q_{1} q_{2} /\left(q_{1}+q_{2}\right)$ and $c(Q)=H_{2}\left(-i q_{1},-i q_{2}\right)$. Furthermore, the last expression in (52) equals the expression

$$
\int_{C_{a, b}[0, T]}^{a n f_{q_{1}}}\left(\int_{C_{a, b}[0, T]}^{a n f_{q_{2}}} F(x+y) d \mu(y)\right) d \mu(x) .
$$

5.3. The Generalized Fourier-Hermite Functional on Function Space. For each $m=0,1,2, \ldots$, and for each $j=1,2, \ldots$, let $H_{m}^{j}(u)$ denote the generalized Hermite polynomial

$$
\begin{aligned}
H_{m}^{j}(u) \equiv & (-1)^{m}(m !)^{-1 / 2}\left(B_{j}\right)^{m / 2} \exp \left\{\frac{\left(u-A_{j}\right)^{2}}{2 B_{j}}\right\} \\
& \times \frac{d^{m}}{d u^{m}}\left(\exp \left\{-\frac{\left(u-A_{j}\right)^{2}}{2 B_{j}}\right\}\right) .
\end{aligned}
$$

Then for each $j=1,2, \ldots$, the set

$$
\left\{\left(2 \pi B_{j}\right)^{-1 / 4} H_{m}^{j}(u) \exp \left\{-\frac{\left(u-A_{j}\right)^{2}}{4 B_{j}}\right\}: m=0,1, \ldots\right\}
$$

is a complete orthonormal set in $L_{2}(\mathbb{R})$. Now we define

$$
\Phi_{\left(m_{1}, \ldots, m_{k}\right)}(x)=\prod_{j=1}^{k} H_{m_{j}}^{j}\left(\left\langle\alpha_{j}, x\right\rangle\right) .
$$

The functionals in (56) are called the generalized FourierHermite functionals. It is known that these functionals form a complete orthonormal set in $L^{2}\left(C_{a, b}[0, T]\right)$; that is to say, let $F \in L^{2}\left(C_{a, b}[0, T]\right)$ and, for $N=1,2, \ldots$, let

$$
F_{N}(x)=\sum_{m_{1}, \ldots, m_{N}=0}^{N} A_{\left(m_{1}, \ldots, m_{N}\right)}^{F} \Phi_{\left(m_{1}, \ldots, m_{N}\right)}(x)
$$


where $A_{\left(m_{1}, \ldots, m_{N}\right)}^{F}$ is the generalized Fourier-Hermite coefficient,

$$
A_{\left(m_{1}, \ldots, m_{N}\right)}^{F} \equiv \int_{C_{a, b}[0, T]} F(x) \Phi_{\left(m_{1}, \ldots, m_{N}\right)}(x) d \mu(x) .
$$

Then

$$
\begin{aligned}
F(x) & =\lim _{N \rightarrow \infty} F_{N}(x) \\
& =\lim _{N \rightarrow \infty} \sum_{m_{1}, \ldots, m_{N}=0}^{N} A_{\left(m_{1}, \ldots, m_{N}\right)}^{F} \Phi_{\left(m_{1}, \ldots, m_{N}\right)}(x)
\end{aligned}
$$

is called the generalized Fourier-Hermite series expansion of $F$. In (59), the limit is taken in the $L^{2}\left(C_{a, b}[0, T]\right)$-sense.

Example 3. Let $q_{0}$ be a nonzero real number and let $\Phi_{\left(m_{1}, \ldots, m_{N}\right)}$ be the generalized Fourier-Hermite functional given by (56) above. Then for all nonzero real number $q$ with $|q| \geq\left|q_{0}\right|$, the modified generalized AFSFI of $\Phi_{\left(m_{1}, \ldots, m_{N}\right)}$ exists and it is given by the formula

$$
\int_{C_{a, b}[0, T]}^{a n f_{q}^{c(q)}, h} \Phi_{\left(m_{1}, \ldots, m_{N}\right)} d \mu(x)=\prod_{j=1}^{N} \int_{C_{a, b}[0, T]}^{a n f_{q}^{c(q)}, h} \phi_{\left(m_{j}, j\right)} d \mu(x),
$$

where

$$
\begin{gathered}
\phi_{\left(m_{j}, j\right)}(x)=H_{m_{j}}^{j}\left(\left\langle\alpha_{j}, x\right\rangle\right) \\
\int_{C_{a, b}[0, T]}^{a n f_{q}^{c(q)}, h} \phi_{\left(m_{j}, j\right)} d \mu(x) \\
=\left(2 \pi B_{j}\right)^{-1 / 2} \int_{R} H_{m}^{j}\left((-i q)^{-1 / 2} u+c(q)\langle\vec{\alpha}, h\rangle\right) \\
\times \exp \left\{-\frac{\left(u-A_{j}\right)^{2}}{2 B_{j}}\right\} d u .
\end{gathered}
$$

The last expression is valid because the generalized Hermite functional is a polynomial with degree $m_{j}$ and hence it has an analytic extension.

Remark 9. Since the set of generalized Fourier-Hermite functionals

$$
\mathscr{M} \equiv\left\{\Phi_{\left(m_{1}, \ldots, m_{k}\right)}\right\}_{k=1}^{\infty}
$$

is a complete orthonormal set in $L^{2}\left(C_{a, b}[0, T]\right)$, we could extend the results for functionals in $L^{2}\left(C_{a, b}[0, T]\right)$ under the appropriate conditions.

\section{Conclusions}

In Section 3, we presented our analysis of the generalized AFSFI and defined the modified generalized AFSFI. Furthermore we explained the physical circumstances with respect to an anharmonic oscillator using the concept of the modified generalized AFSFI. That is to say, we introduced some new concepts in order to explain various physical circumstances. In Section 4, we established some relationships with respect to the modified generalized AFSFI involving the generalized AFSFI; see Theorem 7. Finally, we applied our results to various classes of functionals studied in $[2,4,10,11]$.

\section{Conflict of Interests}

The authors declare that there is no conflict of interests regarding the publication of this paper.

\section{Acknowledgments}

The authors thank the referees for their helpful suggestions which led to the present version of this paper. This research was Basic Science Research Program through the National Research Foundation of Korea (NRF) funded by the Ministry of Science, ICT \& Future Planning (2012R1A1A1004774).

\section{References}

[1] R. H. Cameron and D. A. Storvick, "Feynman integral of variations of functionals," in Gaussian Random Fields, vol. 1 of Series Probability and Mathematical Statistics, pp. 144-157, World Scientific, Singapore, 1991.

[2] R. H. Cameron and D. A. Storvick, "Some Banach algebras of analytic Feynman integrable functionals," in Analytic Functions Kozubnik 1979, vol. 798 of Lecture Notes in Mathematics, pp. 1867, Springer, Berlin, Germany, 1980.

[3] R. H. Cameron and D. A. Storvick, "Analytic Feynman integral solutions of an integral equation related to the Schrödinger equation," Journal d’Analyse Mathématique, vol. 38, no. 1, pp. 3466, 1980.

[4] R. H. Cameron and D. A. Storvick, "Relationships between the Wiener integral and the analytic Feynman integral," Rendiconti del Circolo Matematico di Palermo. Serie II. Supplemento, no. 17, pp. 117-133 (1988), 1987.

[5] K. S. Chang, G. W. Johnson, and D. L. Skoug, "The Feynman integral of quadratic potentials depending on two time variables," Pacific Journal of Mathematics, vol. 122, no. 1, pp. 11-33, 1986.

[6] S. J. Chang, J. G. Choi, and H. S. Chung, "An approach to solution of the Schrödinger equation using Fourier-type functionals," Journal of the Korean Mathematical Society, vol. 50, no. 2, pp. 259-274, 2013.

[7] D. M. Chung and S. Kang, "Conditional Feynman integrals involving indefinite quadratic form," Journal of the Korean Mathematical Society, vol. 31, no. 3, pp. 521-537, 1994.

[8] J. Yeh, "Singularity of Gaussian measures on function spaces induced by Brownian motion processes with non-stationary increments," Illinois Journal of Mathematics, vol. 15, pp. 37-46, 1971.

[9] S. J. Chang and D. M. Chung, "Conditional function space integrals with applications," The Rocky Mountain Journal of Mathematics, vol. 26, no. 1, pp. 37-62, 1996.

[10] S. J. Chang, H. S. Chung, and D. Skoug, "Integral transforms of functionals in $L^{2}\left(C_{a, b}[0, T]\right)$," The Journal of Fourier Analysis and Applications, vol. 15, no. 4, pp. 441-462, 2009. 
[11] S. J. Chang, J. G. Choi, and H. S. Chung, "Generalized analytic Feynman integral via function space integral of bounded cylinder functionals," Bulletin of the Korean Mathematical Society, vol. 48, no. 3, pp. 475-489, 2011.

[12] H. S. Chung, D. Skoug, and S. J. Chang, "Relationships involving transforms and convolutions via the translation theorem," Stochastic Analysis and Applications, vol. 32, no. 2, pp. 348-363, 2014.

[13] E. Nelson, Dynamical Theories of Brownian Motion, Mathematical Notes, Princeton University Press, Princeton, NJ, USA, 2nd edition, 1967.

[14] J. Yeh, Stochastic Processes and the Wiener Integral, Marcel Dekker, New York, NY, USA, 1973.

[15] G. W. Johnson and D. L. Skoug, "Scale-invariant measurability in Wiener space," Pacific Journal of Mathematics, vol. 83, no. 1, pp. 157-176, 1979.

[16] H. S. Chung and V. K. Tuan, "Fourier-type functionals on Wiener space," Bulletin of the Korean Mathematical Society, vol. 49, no. 3, pp. 609-619, 2012.

[17] H. S. Chung, J. G. Choi, and S. J. Chang, "A Fubini theorem on a function space and its applications," Banach Journal of Mathematical Analysis, vol. 7, no. 1, pp. 172-185, 2013. 


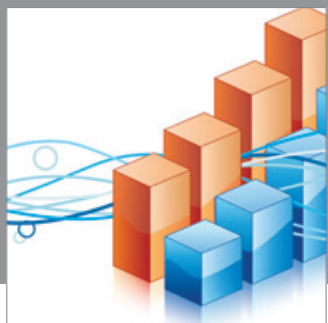

Advances in

Operations Research

mansans

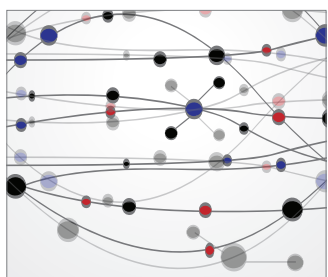

The Scientific World Journal
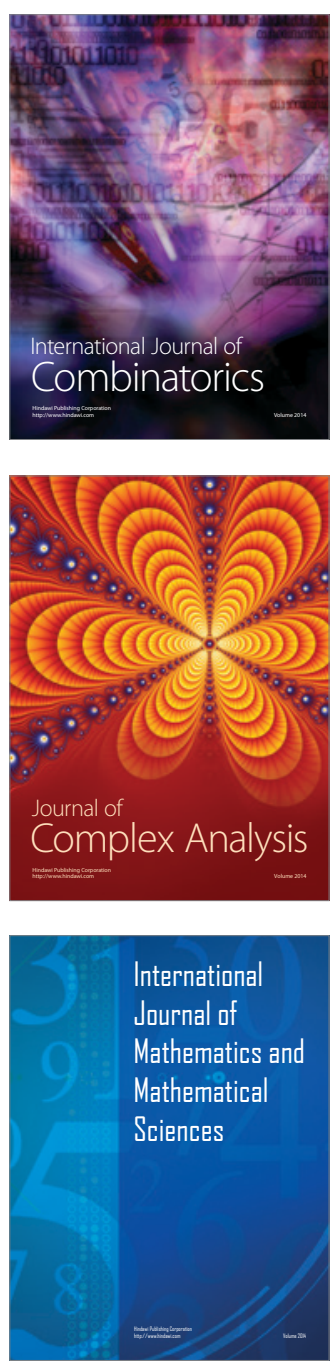
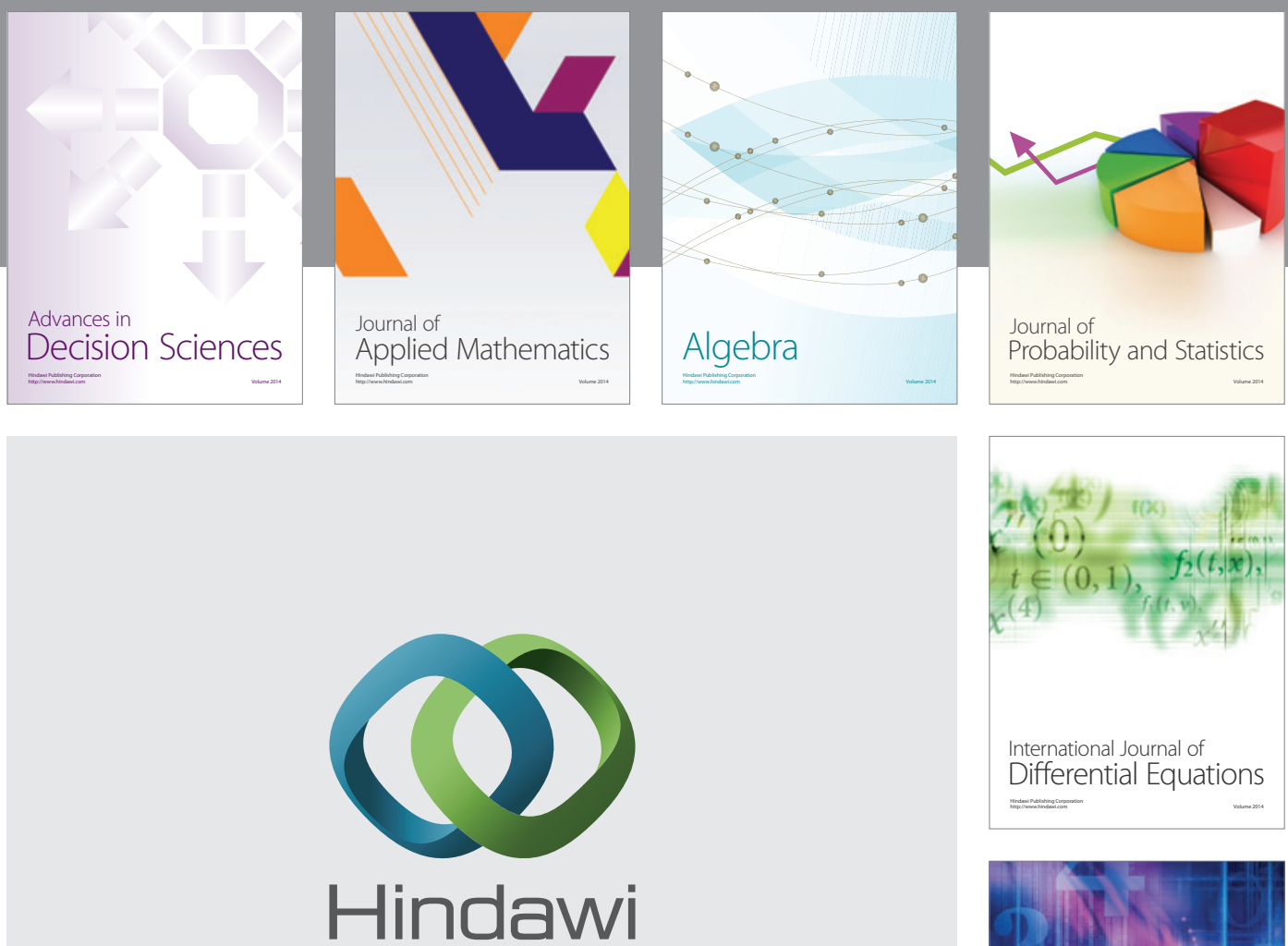

Submit your manuscripts at http://www.hindawi.com
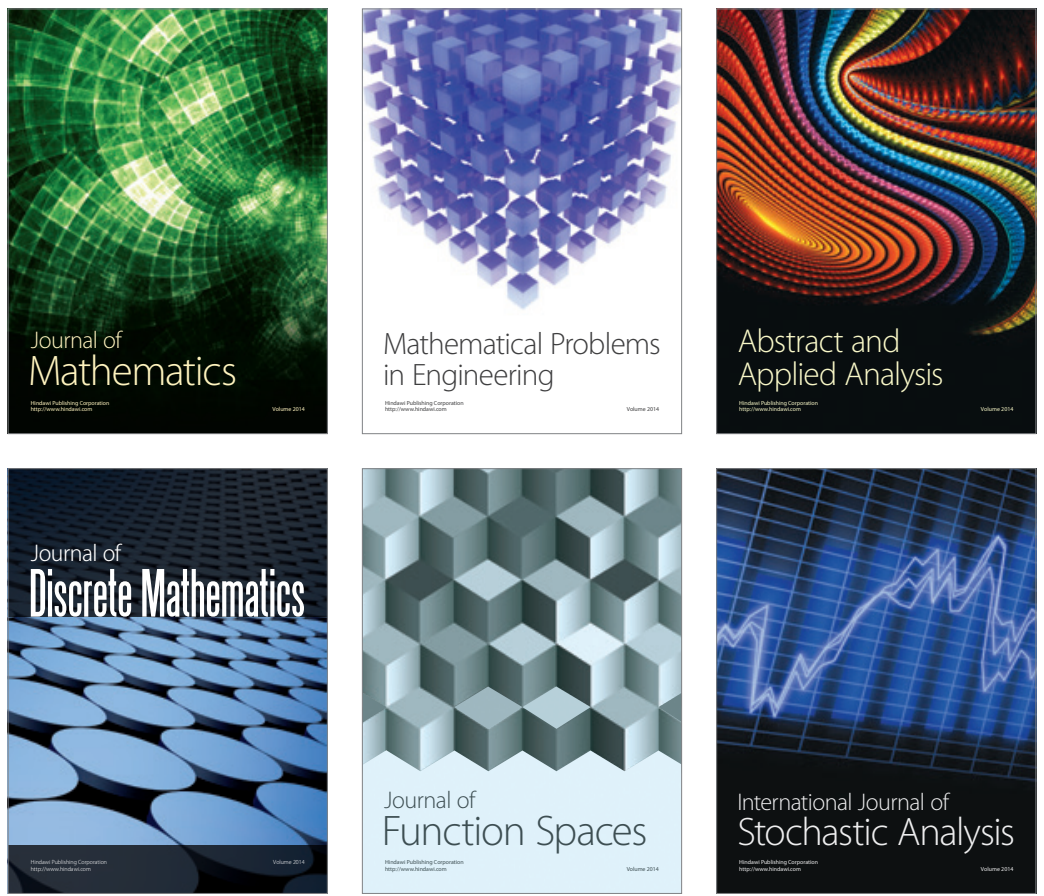

Journal of

Function Spaces

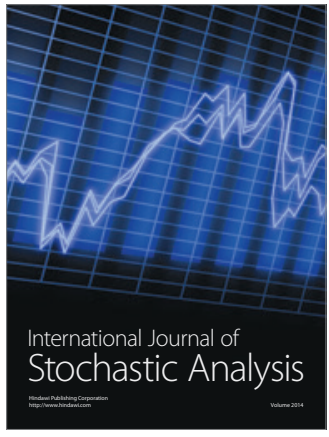

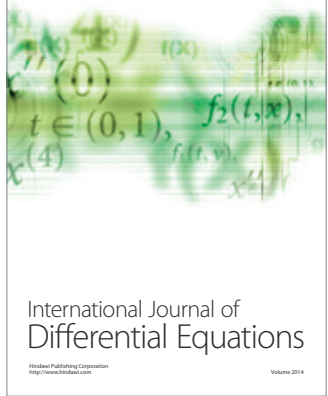
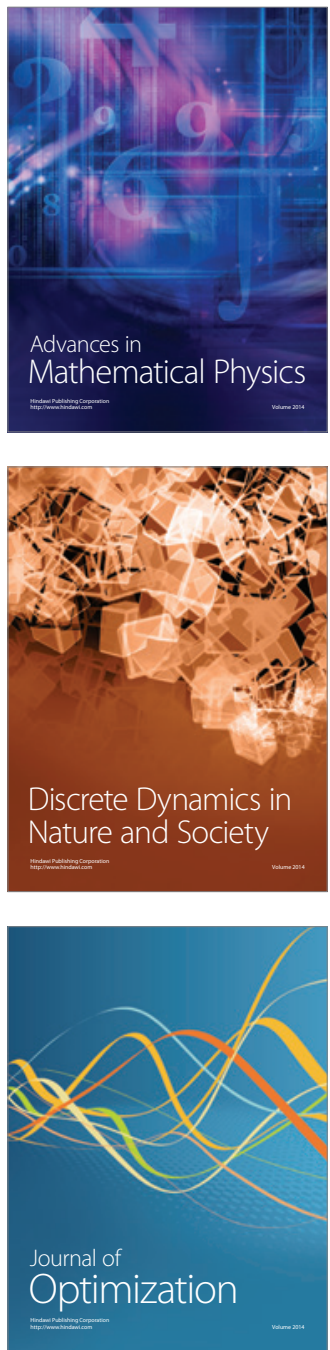